\title{
Aprendizaje basado en proyectos: una propuesta didáctica para el desarrollo socioemocional
}

\author{
Project-Based Learning: A Didactic Proposal for Socio-Emotional \\ Development
}

Jaime Solís-Pinilla ${ }^{1}$

\begin{abstract}
RESUMEN
El presente artículo pretende identificar las etapas del aprendizaje basado en proyectos (ABP) vinculadas al desarrollo socioemocional, por medio de una propuesta de proyecto para estudiantes de secundaria, considerando las nuevas bases curriculares establecidas por el Ministerio de Educación de Chile. Para ello, se analizan distintas dimensiones del aprendizaje socioemocional, la metodología ABP y sus proyecciones en el sistema educacional chileno. Se acompaña lo anterior con una propuesta didáctica basada en el desarrollo de actividades bajo la metodología del $\mathrm{ABP}$, orientada a estudiantes de $3^{\circ}$ y $4^{\circ}$ año de enseñanza secundaria, considerando asignaturas de ciencias naturales. Las nuevas bases curriculares, en transición durante el año 2020, proporcionan espacios idóneos para desarrollar no solamente contenidos, sino también formar a los y las estudiantes de manera integral, desde lo cognitivo, lo social y lo emocional.
\end{abstract}

Palabras claves: aprendizaje socioemocional; aprendizaje basado en proyectos; habilidades para el siglo XXI; educación emocional; reformas curriculares.

\begin{abstract}
This article aims to identify the stages of project-based learning $(P B L)$ related to socio-emotional development, through a project proposal for high school students, considering the new curricular bases established by the Ministry of Education of Chile. To do this, different dimensions of socioemotional learning, the PBL methodology and its projections in the Chilean educational system are analyzed. The above is accompanied by a didactic proposal based on the development of activities under the PBL methodology, aimed at students of 3rd and 4th year of secondary education, considering natural science subjects. The new curricular bases, in transition during 2020, provide ideal spaces to develop not only content, but also train students in a comprehensive way, from the cognitive, social and emotional aspects.
\end{abstract}

Keywords: Socio-emotional learning; project-based learning; 21 st century skills; emotional education; curricular reforms.

\footnotetext{
${ }^{1}$ Profesor de Biología y Ciencias Naturales, Colegio Raimapu -Tierra Florida, La Florida, Santiago de Chile; Magíster en Ciencias Biológicas, Universidad de Chile; Licenciado en Educación, Universidad Metropolitana de Ciencias de la Educación, Santiago, Chile; jaimepinilla87@ gmail.com.
} 


\section{Introducción}

En Chile, durante los últimos 12 meses, la crisis sociopolítica y posterior emergencia sanitaria han develado numerosas falencias en las escuelas, no solo en el ámbito académico, sino también respecto al desarrollo integral del estudiantado, por ejemplo, en cuanto a autoestima, motivación y estado anímico. Lo anterior repercute dramáticamente en un escenario virtual, ante las diversas realidades de nuestro sistema escolar, aumentando las brechas tecnológicas y educacionales. Una de las actuales demandas del sistema escolar, junto con un posible retorno hacia instancias académicas presenciales, es conocer si el estudiantado está aprendiendo en los entornos virtuales de aprendizaje y cómo estos influyen en su desarrollo cognitivo y emocional.

Para Maturana (2001) "las emociones son disposiciones corporales dinámicas que especifican el dominio de acciones de las personas y de los animales” (p.128). Estas han sido motivo de gran preocupación desde la antigüedad (Bisquerra, 2008) y, en nuestras vidas, son un fenómeno muy importante que define el espectro de acciones a realizar, constituyéndose entonces en un aspecto relevante al momento de facilitar los aprendizajes, la apreciación del proceso educativo y las interacciones en al aula (Ibáñez, 2002).

Diversos estudios develan la necesidad de formar competencias socioemocionales, las cuales están relacionadas con un mayor rendimiento académico, sugiriendo que el bienestar socioemocional y los niveles de vínculo con los integrantes de la comunidad influyen en aspectos cognitivos. Las comunidades educativas, entonces, deben ser capaces de generar entornos favorables para el desarrollo de estas competencias (Berger et al., 2014).

Durlak (2011, en Milicic et al., 2013) realizó un metanálisis de numerosos programas de desarrollo socioemocional, evidenciando cómo estos lograban un aumento en índices relacionados con bienestar general, relaciones interpersonales, aprendizaje, compromiso, rendimiento académico y dominio de contenidos, así como una disminución de la sintomatología depresiva. En otras palabras, considerar la formación socioemocional en nuestros/as estudiantes es fundamental para su desarrollo integral. 
En la misma línea, investigaciones respecto a la progresión de indicadores socioemocionales, tales como bienestar socioemocional, autoestima, clima social escolar e integración, fueron desarrolladas por Milicic et al. (2013) para evaluar el Programa para el Bienestar y Aprendizaje Socio Emocional (BASE), financiado por el gobierno de Chile (Fondecyt), analizando su implementación en escuelas de quinto y sexto año de Educación General Básica. Los resultados fueron alentadores, ya que el estudiantado del programa BASE presentó mejoras en autoestima, clima escolar e integración social.

En el contexto de la educación emocional en Chile, Milicic y Marchant (2020) plantean que, si bien hay avances en este campo, como la existencia obligatoria de un Proyecto Educativo Institucional (PEI) por establecimiento, la creación de la Política Nacional de Convivencia Escolar (PNCE), la incorporación de profesionales como encargados/as de convivencia escolar, entre otros, aún hay desafíos para la reducción de índices de violencia, cyberbullying, problemas de clima de aula y desgaste laboral.

Por medio de la Ley 20.370 (Ley General de Educación), publicada en 2009, en Chile se construyó un nuevo marco curricular que culminó en 2019 en las nuevas Bases Curriculares para $3^{\circ}$ y $4^{\circ}$ Medio. Dichas bases aplican tanto para la formación Humanístico-Científica, como Técnico Profesional y Artística. En el artículo 30, se establecen aquellas habilidades, aptitudes y conocimientos que favorecerían un mejor desarrollo del bienestar social y emocional, para formar así futuros/as ciudadanos/as conscientes de su entorno, empáticos/as, con desarrollo moral, autónomos/as, social y medioambientalmente activos/as (Ministerio de Educación [Mineduc], 2019a).

Las nuevas bases curriculares tienen sustento en la necesidad de que los/as nuevos/as ciudadanos/as aprendan y participen en una sociedad de la información que está en constante cambio, proponiendo un grupo de habilidades que les permitan afrontar los desafíos del mundo futuro, llamadas en su conjunto "habilidades para el siglo XXI" (Scott, 2015). Es en este marco curricular, donde el desarrollo de dichas habilidades se articula con una propuesta metodológica conocida como "aprendizaje basado en proyectos" (ABP), entendido este como un método que involucra a los y las estudiantes en su formación, a través de un proceso investigativo compuesto por preguntas auténticas y complejas, acompañado de tareas y productos cuidadosamente diseñados (Cook y Weaver, 2015, en Juanengsih et al., 2018). 
Al considerar estos antecedentes, surge la pregunta: ¿cómo podemos implementar el aprendizaje basado en proyectos y, con ello, desarrollar habilidades socioemocionales? El objetivo de este artículo es identificar las etapas del ABP vinculadas al desarrollo socioemocional, por medio de una propuesta de proyecto para estudiantes de $3^{\circ}$ y $4^{\circ}$ medio, bajo las nuevas bases curriculares de Ciencias Naturales. A partir de esto, se presenta una revisión documental respecto a la metodología ABP, las dimensiones del aprendizaje socioemocional y sus proyecciones en el sistema educacional chileno. Se acompaña lo anterior con una propuesta didáctica, basada en el desarrollo de actividades bajo la metodología $\mathrm{ABP}$, orientada a estudiantes de $3^{\circ}$ y $4^{\circ}$ año de enseñanza secundaria.

\section{Aprendizaje basado en proyectos}

El aprendizaje basado en proyectos (ABP) nace de una propuesta de Kilpatrick en 1918 (en Domènech-Casal, 2018), como una metodología de aprendizaje que consiste en la elaboración de un proyecto a partir de un hecho que provoca interés o curiosidad en el estudiantado (Madueño y Serrano, 2019). Según Cattaneo (2017), la metodología posee un enfoque constructivista al buscar respuestas a las problemáticas planteadas al inicio de cada proyecto, con un enfoque principal en el desarrollo de este.

De forma intrínseca, el estudiantado se motiva a desarrollar cada proyecto y la metodología $\mathrm{ABP}$ releva al contenido como un aspecto más del proceso educativo, junto con la reflexión, la evaluación y la colaboración entre pares. Lo anterior, mediante espacios dialogantes y colaborativos, los cuales, durante el diseño, planificación y ejecución, se discuten constructivamente, ofreciendo sugerencias para su desarrollo (Suparti, 2015, en Santyasa et al., 2020).

El trabajo estudiantil es autónomo y las/os docentes cumplen una labor de supervisión, guía y mediación (Kiraly, 2000, en Carabajal, 2017). La invitación al estudiantado es a buscar fuentes o métodos para obtener respuestas en las fases de desarrollo del proyecto, fomentando habilidades de comunicación y coordinación, en un contexto de aprendizaje inclusivo con sus intereses y habilidades (Soria et al., 2019). 
El ABP promueve habilidades no solo ejecutivas, sino también afectivas (Culclasure et al., 2019), además de habilidades para el siglo XXI. Estas últimas son enmarcadas por la Unesco dentro de cuatro categorías: maneras de pensar, maneras de vivir el mundo, herramientas para trabajar y maneras de trabajar (Scott, 2015). Las habilidades para el siglo XXI buscan la formación de futuros/as ciudadanos/as conscientes de su entorno social y medioambiental, el cual está en permanente cambio, dados los constantes avances tecnológicos y de conocimiento que son propios de la sociedad de la información.

Existen diversos aspectos del ABP que enriquecen a quienes conforman el espacio educativo. Perrenoud (2000) habla de aquellos vinculados a la formación estudiantil, dentro de los que destacan:

a. Movilizar saberes; construir competencias.

b. Visibilizar prácticas sociales que aumentan el sentido de los saberes y de los aprendizajes.

c. Descubrir nuevos saberes, nuevos mundos, en una perspectiva de sensibilización.

d. Generar nuevos aprendizajes en el marco del proyecto.

e. Identificar las adquisiciones y las faltas, en una perspectiva de autoevaluación y de evaluación-balance.

f. Tomar confianza en sí mismo/a, reforzar la identidad personal y colectiva por medio del empoderamiento.

g. Desarrollar la autonomía, la capacidad de elegir y negociar.

Desde la perspectiva docente, el ABP ofrece desafíos interesantes al momento de ejecutarlo, como poseer contenidos y objetivos auténticos, utilizar un sistema de evaluación real, poseer metas explícitas para el estudiantado y crear una situación en donde el profesorado también aprende (Heydrich et al., 2012). 
En el ámbito del ABP en espacios escolares, La Cueva (1998) explicita tres tipos de proyectos, dejando claro que no son excluyentes ni únicos, sino completamente vinculables entre sí:

1. Proyectos científicos: el estudiantado realiza proyectos similares a los que realiza la comunidad científica, bajo las condiciones y alcances que los recursos y contextos puedan abarcar. Ejemplo: comprender el rol de los pigmentos fotosintéticos en la absorción selectiva del espectro lumínico.

2. Proyectos tecnológicos: el estudiantado evalúa o diseña un producto de utilidad práctica, similar a un proyecto de ingeniería. Ejemplo: diseñar mascarillas con diámetros de poro que aumenten la filtración de partículas potencialmente nocivas.

3. Proyectos ciudadanos: los y las estudiantes actúan como sujetos críticos que se sensibilizan, informan y proponen soluciones que pueden difundirse de forma comunitaria. Ejemplos: proponer medidas para el esparcimiento escolar pospandemia; proponer métodos de desalinización de aguas a municipios que presentan escasez de agua potable.

Si bien existe amplia bibliografía respecto de los pasos del ABP, en su esencia, no existe un número concreto de etapas. No obstante, Jalinus et al. (2017) proponen un modelo que clarifica los aspectos troncales dentro del desarrollo de un proyecto:

- Identificación de aprendizajes esperados: se proveen espacios de exploración e información durante el diálogo profesorado-estudiantado, respecto de la relevancia del contenido vinculado al mundo real.

- Comprender el concepto del material didáctico: implica transmitir conocimientos al grupo curso, orientando la búsqueda de material que permita espacios de discusión en la sala de clases.

- Entrenamiento de habilidades: los/as estudiantes, a través de la práctica y demostraciones, aprenden habilidades técnicas, conceptos y procedimientos para llevar a cabo posteriormente las tareas del proyecto. 
- Diseño del proyecto: los/as docentes, en conjunto con el estudiantado, discuten e identifican desafíos o problemáticas del mundo real y cotidiano, de cara a un entorno cambiante que necesita el desarrollo de habilidades para el siglo XXI.

- Propuesta del proyecto: en esta etapa se definen los lineamientos troncales del proyecto, tales como tiempo de producción estimada, lista de materiales, costos asociados, lugar de trabajo.

- Ejecutar las tareas del proyecto: el trabajo colaborativo y la eficiencia en la resolución de problemas son aspectos importantes para considerar en esta etapa. El rol docente se aboca a la supervisión y evaluación del proceso ejecutivo, además de brindar orientación mediante preguntas activas que fomenten la resolución de potenciales problemas.

- Presentación del proyecto: el estudiantado muestra su producto final por medio de un seminario, feria científica, feria del libro u otra instancia extracurricular, con espacios continuos de auto, hetero y coevaluación. 


\section{Figura 1}

Etapas del ABP y roles de docentes y estudiantes, adaptado de Nizwardi, Nabawi y Mardin (2017)

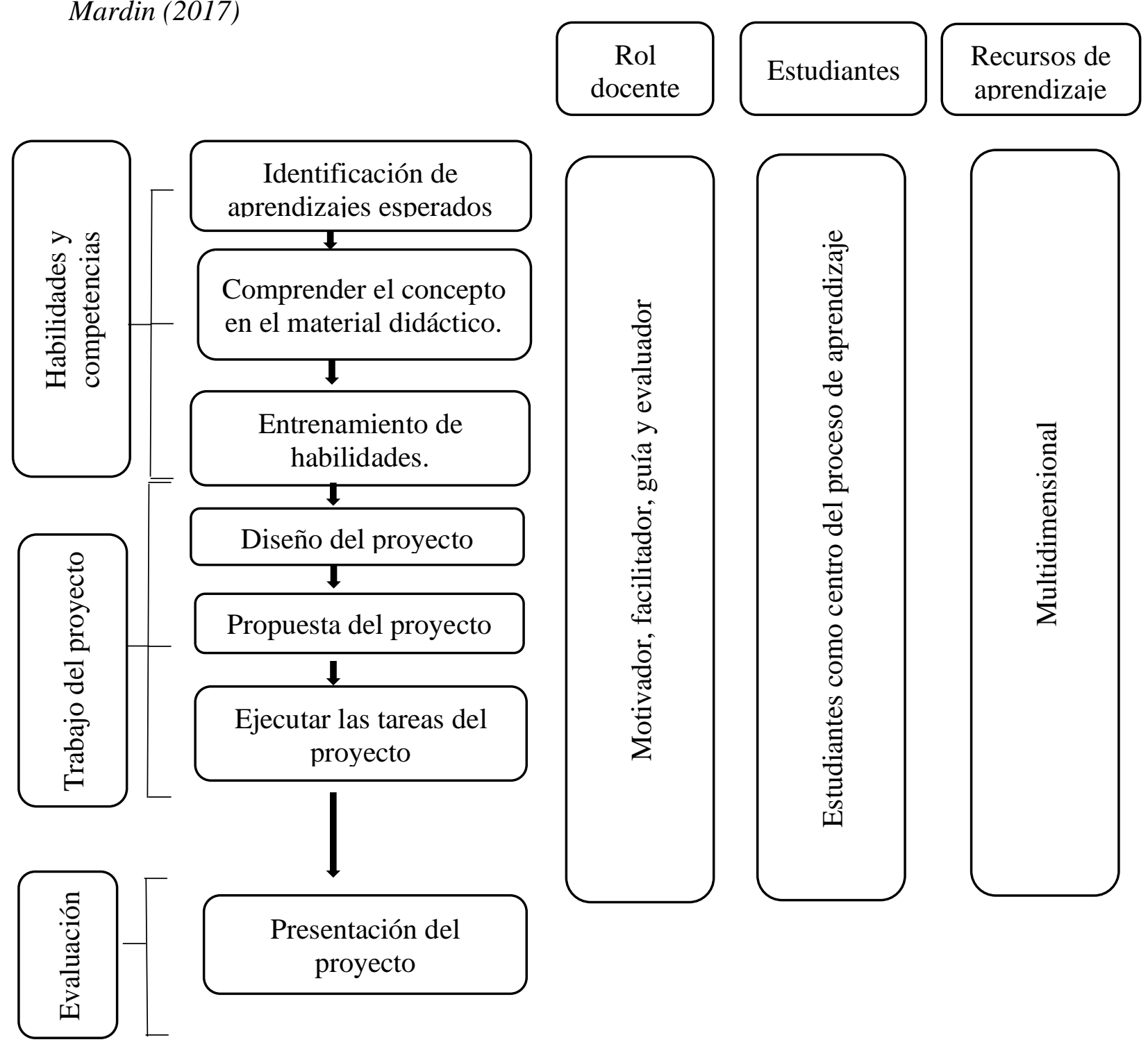

En síntesis, el ABP promueve el desarrollo de habilidades mediante una metodología basada en el desarrollo de un proyecto real, que fomenta el trabajo colaborativo y que, considerando una temática de interés del estudiantado, conlleva una serie de etapas vinculadas a la resolución de problemas, bajo la guía, tutelaje y/o supervisión de los/as docentes involucrados/as. Los y las estudiantes desarrollan habilidades técnicas y sociales, bajo una motivación intrínseca, como principal motor de un proceso de evaluación formativa continua. 


\section{Aprendizaje socioemocional}

En la comunidad educativa, el/la estudiante no solo adquiere formación académica, sino que también establece vínculos con otros miembros de la comunidad y consigo mismo/a, a través del autoconocimiento (Marchant et al., 2013). Esto ha llevado a que las comunidades educativas miren la formación del estudiantado con nuevos ojos, planteándose el propósito de integrar aspectos emocionales y sociales dentro del currículum.

El aprendizaje socioemocional es un proceso donde los individuos manejan sus emociones, establecen metas, muestran y sienten empatía por sus pares, manteniendo relaciones positivas y tomando decisiones responsables (Collaborative for Academic, Social, and Emotional Learning [CASEL], 2020). Dicho proceso "involucra un conjunto de competencias que repercuten directamente sobre su aprendizaje, incluyendo la capacidad de planificación, atención, memoria y metacognición” (Berger et al., 2014, p.628).

De este modo, el aprendizaje socioemocional invita al estudiantado a reconocerse a sí mismo en sus emociones, pensamientos y valores, los cuales influyen en su conducta. De acuerdo a CASEL (2020), se promueven cinco competencias:

1. Conciencia de sí mismo/a: reconocer e identificar nuestras ideas, pensamientos y emociones, y relacionarlas con nuestro comportamiento.

2. Autocontrol: capacidad de regular nuestras emociones, comportamientos o pensamientos.

3. Habilidades relacionales: nos permiten vincularnos mejor con nuestros y nuestras pares.

4. Conciencia social: considerar otros puntos de vista, expresar empatía.

5. Toma responsable de decisiones: considerar las consecuencias que nuestras decisiones tienen sobre nuestros/as pares.

Jennings y Greenberg (2009) proponen un modelo que enfatiza el rol docente para el desarrollo del aprendizaje socioemocional, por medio del cual se establecen vínculos estables en el tiempo entre docente y estudiante. Las clases diseñadas para este objetivo 
se construyen a partir de las fortalezas y habilidades del estudiantado, estableciendo estrategias que estimulen la automotivación, la identificación y resolución de problemas entre grupos de pares, la cooperación y la comunicación en espacios de respeto.

\section{Figura 2}

Modelo de diseño de clases con énfasis en el aprendizaje socioemocional (ASE), adaptado de Jennings y Greenberg (2009)

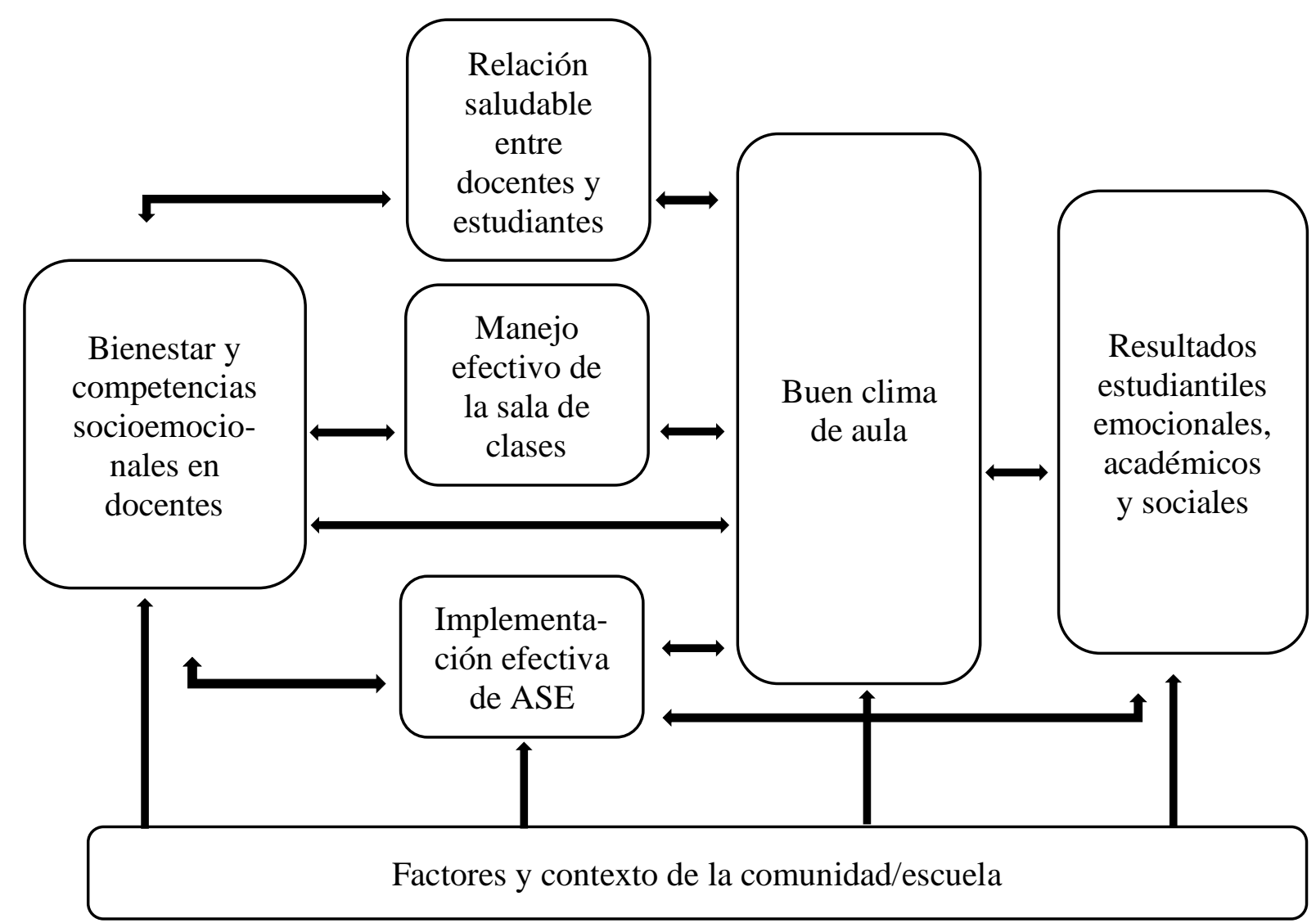

En este modelo, las competencias socioemocionales del cuerpo docente impactan positivamente en el clima del aula, posibilitando que se conviertan en un apoyo efectivo para el estudiantado. Dichas competencias les permiten reconocer las emociones de los/as estudiantes, comprender los componentes cognitivos de sus emociones y asociar estas últimas con la razón en el comportamiento adolescente. 
Según ciertos estudios, las/os docentes que genera un buen clima en el aula se caracterizan por exponer con claridad, reconocer errores como parte del proceso de aprendizaje, destacar progresos, valorar esfuerzos, expresar confianza a sus estudiantes, hacerles sentir importantes y transformar al aula en algo apasionante, desarrollando actividades destacables y atrayentes (Núñez del Río y Fontana, 2009).

De esta manera, los y las docentes que poseen habilidades socioemocionales demuestran una gestión más eficaz del aula y logran usar efectivamente la comunicación verbal y no verbal para motivar al estudiantado, haciendo que disfrute la experiencia de abordar una temática curricular. Asimismo, logran moderar de forma más efectiva los conflictos interpersonales cotidianos, propios de las interacciones humanas.

\section{Una propuesta de desarrollo socioemocional a través del aprendizaje basado en proyectos}

Al considerar los aspectos socioemocionales vinculables a las habilidades para el siglo XXI que propone el ABP, surge la necesidad de implementar proyectos educativos acordes a las nuevas bases curriculares propuestas por el Mineduc (2019b), las que serán parte de las asignaturas de $3^{\circ}$ y $4^{\circ}$ medio desde 2020, en una etapa de transición. En vista de lo anterior, se expone a continuación una propuesta de ABP implementable en estos cursos de educación media, la cual describe aspectos vinculados al desarrollo académico y emocional de los/as estudiantes.

Los y las participantes han de ser estudiantes de $3^{\circ}$ y $4^{\circ}$ medio, quienes pueden elegir tres asignaturas en el plan Humanístico-Científico, de acuerdo a los principios de electividad de las nuevas bases curriculares. A la vez, pueden elegir una o más asignaturas vinculadas a las ciencias naturales, dentro de las que se ofrecen Química, Física, Biología Celular y Molecular, Ciencias de la Salud, y Biología de los Ecosistemas (Mineduc, 2019a).

Para considerar la interdisciplinariedad del proceso, los/as docentes que imparten las distintas asignaturas deben establecer lineamientos y temáticas comunes que permitan abarcar los objetivos de aprendizaje (OA). En vista de lo anterior, una propuesta para 
realizar un proyecto puede ser la temática "Cambio climático y desastres medioambientales", considerando abarcar en cada disciplina los elementos del currículum (Mineduc, 2019a).

Inicialmente, con objeto de concientizar al estudiantado respecto del tema, se generan espacios de diálogo y exploración transversales entre las asignaturas, para reflexionar sobre la importancia del rol que tenemos como ciudadanos/as. Una forma de realizar este primer evento puede ser la creación de espacios de valorización de decisiones, lo que conlleva analizar hechos vinculados al tema, argumentar posturas y considerar los alcances de cada una. Como mediadores/as y guías del proceso formativo, los/as docentes deben generar preguntas motivadoras, tales como "¿debemos seguir avanzando energéticamente como país en base al uso de generadoras a carbón?”, “cómo las plantaciones de eucalipto y pino insigne influyen sobre los ecosistemas?”, “¿cómo influyen las plantas industriales de Ventanas y Quinteros en los ecosistemas costeros?”, “¿cuáles son los principales factores que afectan la disponibilidad de agua dulce en Petorca?”.

Considerando los intereses del estudiantado, debemos incentivar la investigación a partir de la búsqueda de información. Se deben generar instancias que permitan la conversación entre los y las estudiantes, la valorización de su trabajo, el análisis de fuentes bibliográficas y el trabajo colaborativo. Los espacios de reflexión deben abrirse hacia la comprensión de la temática que será abordada a través de un proyecto.

Conforme identifican sus áreas de interés, se forman los grupos de estudiantes que las comparten. Pueden abarcar en un mismo proyecto más de una unidad de aprendizaje, de dos o más asignaturas electivas. Teniendo en cuenta las áreas a abordar, deben considerar un proyecto que sea compatible con todas ellas, el cual pueda ser mostrado a la comunidad por algún medio de transmisión, definiendo así un tipo de proyecto en el que todos los grupos puedan confluir.

Pensando en la afición y destrezas del estudiantado respecto a las redes sociales, puede desarrollarse un ciclo de microdocumentales a ser difundidos mediante estos canales, donde la temática esté vinculada al cambio climático a escala nacional, abarcando distintas áreas de las ciencias naturales y considerando sus objetivos de aprendizaje. 
Teniendo claros los lineamientos desde las distintas perspectivas y aristas del proyecto, los/as integrantes del equipo desarrollan su ejecución, buscando, a modo de ejemplo, la forma en que los precipitados de nitrobenceno e isobutano influyen en el ecosistema marino de Quintero, mediante una propuesta metodológica basada en la indagación histórica de registros bibliográficos de diversas fuentes, tanto gubernamentales como de ONG, relatos de habitantes de la zona, prensa escrita y digital.

Los/as estudiantes, entonces, con la ayuda de su docente guía, se informan y acuerdan en espacios reflexivos, los posibles programas, plataformas de transmisión, formatos y plazos prudentes para realizar este proyecto. De este modo, el estudiantado se informa, practica y adquiere competencias técnicas conforme a los requerimientos.

Apoyar el diseño del proyecto no solo implica que el cuerpo docente trace los lineamientos, sino que además valore las opiniones del estudiantado, realice sugerencias e invite a reflexionar respecto de los alcances y limitaciones del proyecto. Asimismo, debe motivar a investigar y evaluar las implicancias temporales y sociales, así como invitar a considerar distintos puntos de vista de pares, fuentes bibliográficas o docentes especialistas. Generar estos espacios para la reflexión colaborativa entre grupos permite ejercitar la capacidad reflexiva y poner en práctica habilidades para el siglo XXI, en el marco de una coordinación y comunicación eficientes con el entorno. Lo anterior persigue la intención de acompañar a los/as estudiantes en situaciones reales, que les permitan comprender y aplicar lo que están aprendiendo (Flores-Fuentes y Juárez-Ruiz, 2017).

A modo de ejemplo, se pueden encontrar grupos de estudiantes que, al estar en dos electivos como Biología de los Ecosistemas y Química, pueden desarrollar los objetivos de aprendizaje de conocimiento y comprensión (Mineduc, 2019a), los cuales serían, respectivamente: "explicar los efectos del cambio climático sobre la biodiversidad, la productividad biológica y la resiliencia de los ecosistemas, así como sus consecuencias sobre los recursos naturales, las personas y el desarrollo sostenible" (p.183), y "explicar efectos del cambio climático sobre los ciclos biogeoquímicos y los equilibrios químicos que ocurren en los océanos, la atmósfera, las aguas dulces y los suelos, así como sus consecuencias sobre el bienestar de las personas y el desarrollo sostenible” (p.199). 
Tomando en cuenta el enfoque interdisciplinar del nuevo currículum, la presente propuesta conversa con dos disciplinas e incluso más, considerando que el proyecto desemboca en la realización de piezas audiovisuales, las cuales tributan a las asignaturas de Tecnología y Artes, enriqueciendo así el proceso educativo. Al mismo tiempo, se permite que distintos/as docentes conversen con un objetivo común y que se desarrollen habilidades y conocimientos durante la reflexión, en espacios dialogantes conformados por miembros de la comunidad.

Con fines de lograr una mayor reflexión y sensibilización ante los fenómenos de contaminación y cambio climático, es posible girar hacia la transdisciplinariedad, al complementar los microdocumentales con registros sociohistóricos que conversan con la asignatura de Comprensión Histórica del Presente, cuyo objetivo de aprendizaje pertinente es "analizar diversas perspectivas historiográficas sobre procesos de la historia reciente, considerando la importancia del conocimiento histórico en la sociedad y el protagonismo de individuos y grupos en cuanto sujetos históricos y participar en el desarrollo de iniciativas de historia local, recogiendo relatos y fuentes propias de la comunidad cercana para relevar espacios de memoria” (Mineduc, 2019a, p.237).

Cada jornada de búsqueda va acompañada con la supervisión y guía de cada uno/a de los/as docentes involucrados/as y los avances periódicos se van monitoreando mediante evaluaciones formativas, al igual que en las fases anteriores. Es importante consultar las impresiones de los/as estudiantes respecto de lo investigado, sus sentimientos, conocer si empatizan con una postura o relato y, ante posibles conflictos entre opiniones contrarias, invitarlos/as a considerar las perspectivas de otros/as por medio de un diálogo efectivo.

Para finalizar, los microdocumentales resultantes se publican en las redes sociales de la escuela. Esto constituye un evento de cierre concreto del proceso de aprendizaje, no por el mero hecho de la entrega y posterior evaluación, sino porque en esta instancia se evalúa formativa y sumativamente. Se invita a autoevaluarse y coevaluar a los/as pares, motivando instancias reflexivas mediante preguntas desafiantes como "¿qué he podido aprender?”, “¿mi forma de trabajo contribuye a la organización de un proyecto grupal?”, “¿cuáles fueron mis fortalezas y debilidades?” y “¿qué mejoraría de ellas?”. 


\section{Reflexiones finales}

En el siglo XXI se vuelve urgente la implementación curricular del aprendizaje socioemocional. Las competencias socioemocionales permiten a los/as estudiantes conocerse a sí mismos/as y a sus pares, ser más empáticos/as, comprensivos/as y enfrentar de mejor forma los desafíos que la vida les va presentando.

Como docentes, apropiarnos del ABP no es tarea fácil, sobre todo en este período de transición hacia nuevas bases curriculares. Si bien han existido instancias para el conocimiento y formación continua docente, una piedra de tope para la apropiación del $\mathrm{ABP}$ es la rigidez que sigue teniendo el currículum nacional, el cual otorga excesiva relevancia a los contenidos.

El ABP provee espacios de desarrollo personal, enfocados en el autodesarrollo y la gestión de proyectos (Politsinskaya et al., 2019). No obstante, existen estudios que demuestran que también fomenta el desarrollo de habilidades sociales (Cullin et al., 2017; Culclasure et al., 2019), promoviendo un trabajo grupal eficaz, reflexivo y autónomo, mediante el cual el estudiantado da sentido a su proceso de aprendizaje (Kokotsaki et al. 2016). Estos estudios avalan la efectividad del método ABP en aulas de distintos niveles educativos. Sin embargo, la invitación es a reflexionar si el currículum está preparado para fomentar dichas habilidades, restar relevancia a las calificaciones, enfocarse en el impacto que la educación socioemocional puede generar en el desarrollo estudiantil y cambiar los paradigmas docentes respecto a la forma en que se concibe la educación en las aulas.

Tanto en la educación socioemocional como en el desarrollo de un proyecto mediante el ABP, el rol docente es relevante y su labor está enfocada a orientar, moderar y facilitar el desarrollo de cada etapa (Carabajal, 2017). No obstante, ¿cuántos de aquellos roles ya los realizamos en el aula?, ¿cuál es el enfoque que debiéramos brindar en este tipo de proyectos?, ¿somos docentes que propiciamos la reflexión o, más bien, nos dedicamos a dar lineamientos que para muchos/as no tienen sentido? En términos de desarrollo emocional, ¿nos conocemos lo suficiente como para orientar el desarrollo de nuestros y nuestras estudiantes? 
Por último, es importante analizar la forma en que se ha dado la implementación de las nuevas bases curriculares bajo el contexto social y sanitario actual, revisar cómo se ha acompañado a los/as docentes en este proceso e identificar las posibles complicaciones que la escuela a distancia ha generado a este respecto. Considerar el ABP como potencial catalizador de las habilidades para el siglo XXI y el desarrollo emocional estudiantil, plantea nuevos desafíos que invitan a ampliar estas prácticas hacia cursos de niveles menores y, con ello, generar nuevas líneas de investigación que evalúen su progresión, resultados y limitaciones dentro del sistema educacional chileno.

\section{Referencias}

Berger, C., Álamos, P., Milicic, N. y Alcalay, L. (2014). Rendimiento académico y las dimensiones personal y contextual del aprendizaje socioemocional: evidencias de su asociación en estudiantes chilenos. Universitas Psychologica, 13(2), 627-638. http://dx.doi.org/10.11144/Javeriana.UPSY13-2.radp

Bisquerra, R. (2008). Educación socioemocional y bienestar. Wolkers Kluwer.

Carabajal, R. (2017). Propuesta didáctica para abordar la Traducción Técnica: trabajo colaborativo y aprendizaje basado en proyectos. Revista Digital de Investigación en Docencia Universitaria, 11(2), 192-202. http://dx.doi.org/10.19083/ridu.11.571

Collaborative for Academic, Social, and Emotional Learning. (2020). Beneficios de SEL versión en español. Recuperado de https://casel.org/what-is-sel/

Cattaneo, K. (2017). Telling Active Learning Pedagogies Apart: From Theory to Practice. Journal of New Approaches in Educational Research (NAER Journal), 6(2), 144152. https://doi.org/10.7821/naer.2017.7.237

Culclasure, B., Longest, K. y Terry, T. (2019). Project-Based Learning (Pjbl) in Three Southeastern Public Schools: Academic, Behavioral, and Social-Emotional 
Outcomes. Interdisciplinary Journal of Problem-Based Learning, 13(2). https://doi.org/10.7771/1541-5015.1842

Cullin, M., Hailu, G., Kupilik, M. y Petersen, T. (2017). The Effect of an Open-Ended Design Experience on Student Achievement in an Engineering Laboratory Course. International Journal of Engineering Pedagogy (iJEP), 7(4), 102-116. https://doi.org/10.3991/ijep.v7i4.7328

Domènech-Casal, J. (2018). Concepciones de alumnado de secundaria sobre energía. Una experiencia de aprendizaje basado en proyectos con globos aerostáticos. Enseñanza de las ciencias, 36(2), 191-213. https://doi.org/10.5565/rev/ensciencias.2462

Flores-Fuentes, G. y Juárez-Ruiz, E. (2017). Aprendizaje basado en proyectos para el desarrollo de competencias matemáticas en Bachillerato. Revista Electrónica de Investigación Educativa, 19(3), 71-91. https://doi.org/10.24320/redie.2017.19.3.721

Heydrich, M., Rojas, M. y Hernández, A. (2012). Aprendizaje basado en proyectos: una experiencia de innovación docente. Revista Universidad EAFIT, 46(158), 11-21.

Ibáñez N. (2018). Las emociones en el aula. Estudios Pedagógicos, (28), 31-45. https://doi.org/10.4067/S0718-07052002000100002

Jalinus, N., Nabawi, R. y Mardin, A. (2017). The Seven Steps of Project-Based Learning Model to Enhance Productive Competences of Vocational Students. International Conference on Technology and Vocational Teachers (ICTVT 2017). Atlantis Press.

Jennings, P. y Greenberg, M. (2009). The Prosocial Classroom: Teacher Cocial and Emotional Competence in Relation to Student and Classroom Outcomes. Review of Educational Research, 79(1), 491-525.

Juanengsih, N., Mahmudah, L. y Ruri, R. (2018). Enhancing of Students' Learning Outcomes in the Environment Pollution Concept Through Project Based Learning 
(PjBL). Jurnal Penelitian dan Pembelajaran IPA, 4(2), 127-134. https://doi.org/10.30870/jppi.v4i2.3385

Kokotsaki, D., Menzies, V. y Wiggins, A. (2016). Project-based Learning: A Review of the Literature. Improving Schools, 19(3). 267-277. https://doi.org/10.1177/1365480216659733

La Cueva, A. (1998). La enseñanza por proyectos: ¿mito o reto? Iberoamericana de Educación, (16), 165-187.

Madueño, G. y Serrano, R. (2019). La ciudad como contexto educativo: desarrollo de la competencia cívica en las aulas infantiles y en el alumnado con necesidades de apoyo educativo. Foro de Educación, 17(27), 203-227. https://doi.org/10.14516/fde.649

Marchant, T., Milicic, N., y Álamos, P. (2013). Impacto en los niños de un programa de desarrollo socioemocional en dos colegios vulnerables en Chile. RIEE. Revista Iberoamericana de Evaluación Educativa, 6(2), 167-186.

Maturana, H. (2001). Cognição, Ciência e Vida Cotidiana. Editorial UFMG.

Milicic, N. y Alcalay, L. (2020). Educación Emocional en el sistema escolar chileno: un desafío pendiente. En M. Corvera y G. Muñoz. Horizontes y propuestas para transformar el sistema educativo chileno (pp. 53-62). Ediciones Biblioteca del Congreso Nacional de Chile.

Milicic, N., Alcalay, L., Berger, C., y Álamos, P. (2013). Aprendizaje socioemocional en estudiantes de quinto y sexto grado: presentación y evaluación de impacto del programa BASE. Ensaio: Avaliação e Políticas Públicas em Educação, 21(81), 645-666. https://doi.org/10.1590/S0104-40362013000400002.

Ministerio de Educación de Chile (2019a). Bases Curriculares $3^{o}$ y $4^{o}$ medio. https://www.curriculumnacional.cl/portal/Secciones/3-y-4-medio/133992:3-y-4medio-Nuevo-Curriculum

Ministerio de Educación de Chile (2019b). Metodología de Aprendizaje Basado en Proyectos. https://www.curriculumnacional.cl/portal/Innovacion/Lineas-de- 
Innovacion/STEM-Aprendizaje-Basado-en-Proyecto-ABP/140166:Metodologiade-aprendizaje-basado-en-proyectos

Núñez del Río, M. y Fontana, M. (2009). Competencia socioemocional en el aula: características del profesor que favorecen la motivación por el aprendizaje en alumnos de Enseñanza Secundaria Obligatoria. Revista Española de Orientación y Psicopedagogía 20(3), 257-269.

Perrenoud, P. (2000). Aprender en la escuela a través de proyectos: ¿Por qué? ¿Cómo? Revista de Tecnología Educativa,14(3), 311-321.

Politsinskaya, E., Lizunkov, V. y Ergunova, O. (2019). Organization of student projectbased activities through individual learning routes. International Journal of Emerging Technologies in Learning (iJET), 14(11), 186-193. https://doi.org/10.3991/ijet.v14i11.10312

Santyasa, I., Rapi, N. y Sara, I. (2020). Project Based Learning and Academic Procrastination of Students in Learning Physics. International Journal of Instruction, 13(1), 489-508. https://doi.org/10.29333/iji.2020.13132a

Scott, C. (2015). El futuro del aprendizaje 2 ¿Qué tipo de aprendizaje se necesita en el siglo XXI? Unesco. https://unesdoc.unesco.org/ark:/48223/pf0000242996_spa

Soria, I., Gómez, C., Monsalve, B. y Fontanillo, A. (2019). Aprendizaje cooperativo basado en proyectos y entornos virtuales para la formación de futuros maestros. Educar, 55(2), 519-541. https://doi.org/10.5565/rev/educar.935 